\title{
Design on routing optimization algorithm based on complex network
}

\author{
Yang GUO, Jinhe, ZHOU \\ (school of Informations Communication Engineering,Beijing Information Science and Technilogy University,Beijing 100101，China)
}

\begin{abstract}
In order to solve the problem of information flow network transmission and congestion control in complex internet networks,A new and complicated routing network algorithm based on network evolution simulation model is proposed,An improved method is proposed for a typical static local routing algorithm in an SRTD network. This method adds a node message queue length as a main parameter. The variable parameters can be used to adjust the node processing capacity and the weight of node packet queue length in the routing policy. And to a certain extent, the efficiency of simulation is improved and the purpose of network optimization is achieved.
\end{abstract}

\section{Introduction}

In real life, the application of complex networks in the information society is more and more frequent. In network complexes, the most common phenomenon is network congestion. Large areas of network congestion can cause network performance to crash. At this time, an effective strategic route is needed to avoid network congestion and improve performance by accessing local network routers through data packets. Incorrect planning routes and unknown factors may increase network congestion and even cause network [1] collapse. The research results of the researchers are that the network has started to study network congestion control from a homogeneous network, but in practice, many of them are scale-free. Therefore, research on scale-free networks emphasizes the focus of research.

Watts and Strogatz [2] proposed the WS small-world model. Shortly after the same century, Barabási and Albert [3] studied the complex network and proposed a scale-free network model. The proposed two models set off research on complex networks. upsurge. They think that the complex network in the real world is dynamically evolving. The phenomenon of scale-free reality networks comes from these two factors: the growth mechanism and the preferential connection mechanism. In the small world experiment of Milgram [4], people used a simple decentralized algorithm to search for target objects, namely "greedy algorithm". Because this algorithm itself is very simple and has no special nature, there is a certain number of letters successfully reaching the target within a short number of steps. This fact shows that the social network structure itself has a certain degree of particularity. In early 2000, Kleinberg [5] first applied the grid method to research and prove the search capabilities of complex networks, confirming that a fast search can be implemented in complex networks. Watts [6] made further research on the issue of search capabilities. After

\footnotetext{
a Corresponding author: 18811448607@163.com
}

the searchability of complex networks was confirmed, a large number of search algorithms became the stage for the study of complex networks.

In fact, each routing strategy has limitations, so we require a lot of experimentation to get a specific environment.Better performance routing policy. In the near future, there must be some redundant information and high transmission efficiency.Strong anti-congestion routing strategies were discovered and used by us.

\section{Introduction to basic knowledge}

\subsection{Status of Network Routing}

One of the main contents of this study is that there are no rules, The strategy includes the network route. The strategy of the existing route, ie shorter route and fewer routes and strategies The motivation for this paper comes from setting up a SRID (Setting Up Routing Table Dynamically).

In the network, the hypothesis $\mathrm{R}$ of the packet data node generated at each time interval, then the probability of $\mathrm{R}$ is $N / N-1$. At the center of this definition, node $\mathrm{B}$ passes the number of nodes in all paths. Therefore, after the packet, such as $R B / N(N-1)$. C's physical capacity to ensure that the network's mobility reaches $R B / N(N-1)>C$. Assume the red model, $\mathrm{C}=1$. Then the rate at which packets are $R B / N(N-1)>C$ is the largest. And R, in some cases, depends on B max. The $B_{\max }$ smaller the size, the larger $\mathrm{R}$ is, and the later the network congestion occurs. The effect of such network simulation is better. Therefore, on the road to improving routing strategies, the central idea of this article is to reduce the number of networks in the center of the most valuable node network. By reducing the maximum centered value, postponing network congestion and 
increasing network delivery rate, [7] loss of lost packets is reduced.

\subsection{Existing Routing Policy}

\subsubsection{Shortest Path Routing Policy (SPEA)}

When the path strategy is applied to the shortest path, use the Floyd[8] algorithm. Freud's algorithm, the strategy between weighted vertices, looks for shorter paths. The process algorithm is as follows:

1) From any unilateral road. The distance between two points is all the right border, if it is not connected to the edge between two points, the distance is infinite.

2) For each vertex $a$ and $b$, we need to see if there is a c vertex, which is the optimal path from the shortest path from a to b. If so, update it.

\subsubsection{Degree and Minimum Routing Policy}

The core idea of this strategy is [9], in the process of creating a routing table, find the minimum path of all nodes to avoid the possibility of maximum congestion. Therefore, it can reduce the degree of centrality of larger problems, reduce the value of central time, postpone the delivery of congestion, and reduce the rate of congestion to postpone the emergence of network congestion.

\subsubsection{Creating Route Table Routing Policies Dynamically}

The core concept of this strategy is that the first is to directly connect the nodes and no longer find the path so that the routing table can be directly written and then the centrality of the nodes can be changed. So, from the first node, node and center to the minimum. Whenever two nodes find a path, they should be recorded in the routing table, plus 1 in the center between them. Repeat the previous process until you find all the paths.

In the application of strategy, finding the path between every two nodes is in the Dijkstra [10] algorithm. The Dijkstra algorithm is used to compute all the nodes that have the shortest route from the node to another node. Its calculation structure is as follows:

1) For each point, take an initial value $L(I)$ from the initial distance of I. L $(S)=0$, other values are inf.

2) All points are marked as unvisited. Point $\mathrm{c}$ is the current source.

3) At point $C$, the distance value of $J$ is updated taking into account all points that are not related to $\mathrm{j}$.

$\mathrm{L}(\mathrm{j})=\min (\mathrm{L}(\mathrm{j}), \mathrm{L}(\mathrm{c})+\mathrm{P}(\mathrm{c}, \mathrm{j}))$

4) The current point visited by marker C. L (c) is the shortest point of $\mathrm{c}$. If all the points have not been visited yet. Otherwise, no. In the interview with the minimum point, its value is not the same as the current point $\mathrm{C}$, so continue to operate in step 3 .

If you find a lesser route between two points, the length of the route and the serial number are on the nodes of the route it passes through. If there is no path, the program will ask "The inf is infeasible!" On the strategic route, considering two different types of nodes, it is not a variable. At the highest level of the center and the node, this may result in a packet path. When most people avoid these nodes, the nodes are relatively free. So this algorithm changes the network node, the closest central node. Therefore, the largest value center can also reduce the performance of the network and can also improve.

\section{Network system model and route optimization strategy}

The scale-free network model is closer to reality. Therefore, strategically, choose to study the evolutionary model of scale-free networks for routing strategy analysis [11].

\subsection{Network Features}

There are three measurement characteristics of network indicators [12]:

1) Average path. In the network, the shortest path number between two points it contains is defined as the distance between two points, and the average distance of the network is a medium-distance node. Formulated as follow:

$$
\mathrm{d}=\frac{1}{N(N-1)} \sum_{i \neq j} d_{i j}
$$

$\mathrm{d}_{\mathrm{ij}}$ is the shortest path connecting node I and node $\mathrm{j}$.

2) Cluster coefficient. For some nodes, adjacent nodes, even in all possible proportions, are defined as the cluster coefficients of the network, which is the average of the coefficients on the nodes. The formula is used as follows:

$$
C=\frac{1}{N} \sum_{i} c_{i}
$$

3) Degree distribution: The distribution of degrees refers to the number of node connections between nodes and nodes of the network. The formula is used as follows:

$$
k_{i}=\sum a_{i}
$$

The probability that the degree of the network is distributed in the node of the network in $\mathrm{k}$ is the law that the degree $\mathrm{k}$ of the node changes. The distribution of the degree in the actual network represents the regularity of the power function:

$$
p_{k}=\frac{1}{N} n_{k}
$$
$\mathrm{k}$

Where $n_{k}$ represents the number of nodes with degree

4) Delivery rate. In the case of a set of encapsulation rates, the percentage of data transferred successfully accounts for the total number of data packages.

5) Congestion. The definition of congestion is represented by the following equation:

$$
H(R)=\lim _{t \rightarrow \infty} \frac{C}{V} \frac{\langle\Delta W\rangle}{\Delta t}
$$

Where: $\Delta \mathrm{W}=\mathrm{W}(\mathrm{t}+\Delta \mathrm{t})-\mathrm{W}(\mathrm{t}), \mathrm{W}(\mathrm{t})$ is the number in the t-time network. According to the number of packets, $\mathrm{V}$ is the speed of dispatch, and $\mathrm{C}$ is the ability to process the data of the node. 


\subsection{Network System Model}

Here is a detailed introduction to the network simulation model. With this simulation in the model, in a period of time, the entire system generates $\mathrm{R}$ packets. Randomly select the source and end nodes of the packet. Assuming that the node's transfer is the same as the location data package, ie the static root table, all points in each time slot are $C$ 's packets, $\mathrm{C}=1$. A data arrives at the destination, and the entire system does not consider that. The concern here is the critical value $R_{c}$ of the transmission of system information along with the chaos of circulation. The critical value is exactly the opposite. Full simulation system handles the maximum capacity of data packages. In general, $\mathrm{R}<R_{c}$, that is, the number of packets generated by the system is smaller than that when the pressure occurs. The amount of packaging for the quantity, generated, and transmitted data is balanced so that it can be. Transfer data stably, keeping the system as a whole smooth. If $\mathrm{R}>R_{c}$ increases with the data packets, the traffic is very congested.

\subsection{Optimization Strategy}

By analyzing the functions of each module of the complex network simulation platform, we find that: In terms of network model construction, the simulation platform only provides a SRTD network model, and there are many kinds of real-world network models. In order to more fully reflect the actual network, this paper implements network models such as Lattice, Stochastic, Stochastic, NW, and WS Small World under the simulation platform based on the construction algorithm of the complex network. In addition, in terms of simulation experiments, the simulation is performed. The platform lacks a visual user interface. Using MATLAB, a user-friendly test interface is built. You can use the interface to select the network model to be set up and set the parameters needed during the simulation.

Therefore, an improved method is proposed for a typical static local routing algorithm in an SRTD network. This method adds a node message queue length as a main parameter, and can adjust the node processing capacity and the node message queue length through variable parameters. The weight in the routing policy. The simulation experiment in the SRTD network model shows:

1) When the parameter $a=3$, the network performance is optimal;

2)Due to the increase of the dynamic parameter of the queue length of the node message, the routing algorithm can more accurately determine the congestion status of the node and determine the forwarding path. Therefore, the improved routing strategy is superior to the classical static local routing strategy in improving the network performance.

\section{Simulation and result analysis}

Network simulation In the case of a certain starting speed, the simulated data transmission process counts the number of successfully delivered data packets and the degree of network congestion. Based on the scale-free network model, according to the MATLAB tool, shortest path channel strategy (SPFA), degree and minimum channel strategy and dynamic route table channel strategy (SRTD) to establish the network simulation, and then go to the perspective of delivery rate congestion. The performance of these three strategies was compared.The initial conditions for the simulation of the network are: 3 initial nodes, each time the added node is connected to 3 sides, and the final size of the node is 100 . The simulation results of the three route strategies are compared: the route of the shortest route, the strategy of the centrality and the minimum route, and the maximum center of the dynamic route. The changes in the maximum centrality are shown in Table 1.

Table 1. Maximm centrality change data table of different routing strategies.

\begin{tabular}{|c|lll|}
\hline Routing strategy & \multicolumn{3}{|c|}{ Maximum centrality } \\
\hline Shortest path routing strategy & 1972 & 2086 & 2948 \\
\hline $\begin{array}{c}\text { Degree and minimum routing } \\
\text { strategy }\end{array}$ & 842 & 898 & 1162 \\
\hline $\begin{array}{c}\text { Dynamically establishing } \\
\text { routing policy for routing table }\end{array}$ & 660 & 665 & 801 \\
\hline
\end{tabular}

The average path length of the shortest path route is 2.661, the smallest degree; the average path of the route strategy is 3.199 , and the route strategy of the road surface is dynamically established. The length of the average path is 3.305 .

In Figure 4, the blue line represents the strategy of the shortest path, the red line represents the degree and the trail is based on strategy, and black is dynamic, establishing the route of the route table.

1) Analysis of Figure 4 can improve the simulation effect of the root strategy. Compared with the route strategy of the route, there has been a certain improvement. Looking at the delivery rate, there was no traffic jam in the initial network. According to the package, the delivery rate was relatively high and the delivery rate was relatively expensive. Together with the increase in the rate of contracting, the largest node in the center became chaotic. Then, the phenomenon of species rapidly spread across the entire network, and the network began to congest and began to distribute. The proportion has also begun to decline. The strategy with the shortest path has the fastest delivery rate, and the strategy with the degree is the minimum and the route is the best. The best is to dynamically establish the route of the route as the strategy. From the standpoint of congestion, if the packet has 3 packets/time, the shortest path route strategy begins to get confused. This is a strategy of a degree and a minimal route. 12 packs/time came out and began to crowd. It's best to be dynamic. According to the strategy of the table route, when the packet rate reaches 16 packets/time, packet loss begins. 
2)Analysis of Figure 1, Figure 2, Figure 3, the performance of the network and the distribution of the central part of the node have a certain relationship. The centrality of the strategy for the shortest path is obviously that the distribution is obviously uneven, and some nodes are very busy and are prone to congestion. We have to establish a route strategy and the distribution of the strategic points of the route is relatively uniform, and the data that each node is responsible for will bear the task load. Because the volume is quite large, it will definitely be delayed when it is crowded, and it has been improved to some extent.

3) Analyze Table 1 to see the performance of the network and the maximum center value. There is a certain relationship. The smaller the maximum center value, the better the network performance.

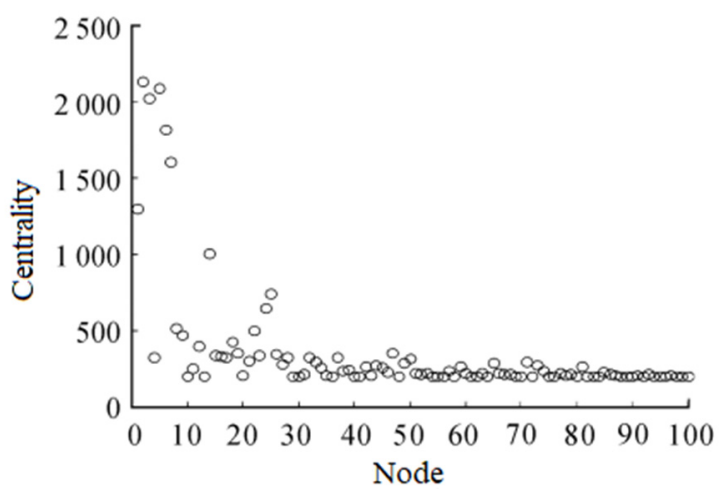

Figure 1. Distribution of the centrality of the shortest path routing strategy node.

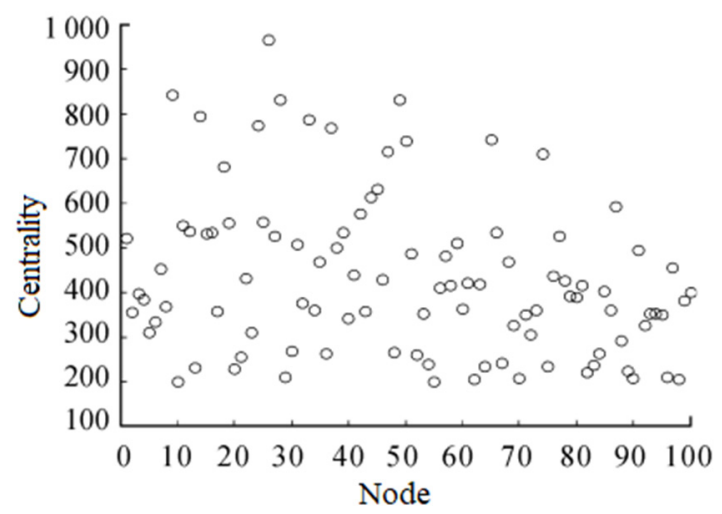

Figure 2. Degree and Minimum Routing Policy Node Centrality Distribution.



Figure 3. Dynamic establishment of routing table strategy node centrality distribution.

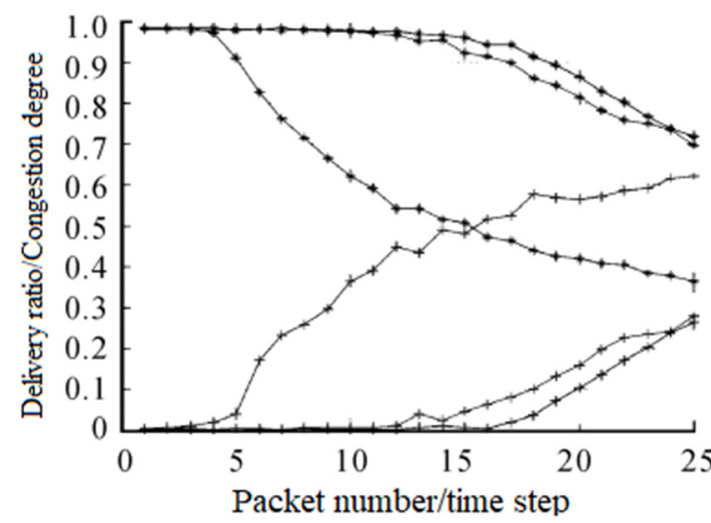

Figure 4. Different routing strategy network simulation results.

\section{Conclusion}

In order to solve the traffic congestion problem of networks existing in complex networks and reduce the network to improve data transmission efficiency, a sophisticated network path strategy SRTD is optimized here. Based on the scale-free network model, a simulation experiment is conducted on the network. From the perspectives of delivery rate and congestion degree, performance comparisons were performed.The simulation results show that the network simulation results of SRTD can improve the network data flow comparable to the existing route strategy to a certain degree. It is in line with the future needs and can effectively solve the congestion control and achieve the purpose of network optimization.

\section{References}

1. R. Franke, G. Lvanova. FALCON or how to compute measures time efficiently on dynamically evolving dense complex networks[J]. Journal of Biomedical Informatics, 2014, 47: 62-70 .

2. Jin Tang, Bo Jiang, Chin-Chen Chang, Bin Luo. Graph structure analysis based on complex network[J]. Digital Signal Processing, 2012, 5:713725 .

3. Wang Lin, Zhang Shuyan. Evolutionary Analysis of Internet AS Network[J]. Journal of Systems Engineering, 2010 (01).

4. Erds P, Rényi A. On the evolution of random graphs[J]. Publications of the Mathematical Institute of the Hungarian Academy of Sciences, 1960, 5: 1761.

5. Adamic L A, Adar E. Friends and neighbors on the web [J]. Social networks, 2003,

6. Watts D J, Dodds P.S., and Newman M,E,J , Identity and Search in Social Network[J].Science, 2002,296:1302-1305.

7. Shi Dinghua. Talking from the geometric growth network[J]. Complex Systems and Complexity Science, 2010, 7(2) : 1-8. 
8. Wang Xianpeng. Research on routing strategies on complex networks[D]. Shanghai: Shanghai Jiaotong University, 2009.

9. Fang Jinqing, Wang Xiaofan, Liu Zengrong. On complexity issues and research on nonlinear complex network systems[M]. Science and Technology Review, 2004(2): 9-12.

10. Tang M, Zhou T.Efficient routing strategies in scalefree networks with limited bandwidth $[\mathrm{J}]$. Physical review E,2011,84(2) : 2-4.

11. Bogdan D, Yu Y, John A. Optimal transport on complex networks $[\mathrm{J}]$. Physical review E,2006,74: 1-3.

12. Yan G, Zhou T, Hu B. Efficient routing on Complex Networks[J].Physical review E, 2006(73) : 1-5. 